\title{
La privacidad, un desafío para periodistas y comunicadores. Análisis de la privacidad en los estudios universitarios de comunicación de Europa y Estados Unidos
}

\author{
Privacy, a challenge for journalists and communicators. \\ Analysis of privacy in university communication studies of Europe and the \\ United States
}

\author{
Dra. Laura Cervi \\ Universidad Autónoma de Barcelona | Carrer de la Vinya, 738, 08193, Cerdanyola del Vallès, \\ Barcelona | http://orcid.org/0000-0002-0376-0609 | laura.cervi@uab.cat

\section{Dr. Carles Marín-Lladó} \\ Universidad Rey Juan Carlos | Camino del Molino, s/n, 28943, Fuenlabrada, Madrid | \\ http://orcid.org/0000-0001-7456-5889 | carles.marin@urjc.es
}

Fechas | Recepción: 07/10/2021 | Aceptación: 01/12/2021

\section{Resumen}

El derecho a la privacidad del ciudadano y su preocupación por la protección de sus datos se ha acentuado con la consolidación y el uso masivo de internet, donde se ven expuestas, cada vez más, millones de referencias personales tanto de usuarios directos como de navegantes esporádicos.

Pero si hay una profesión que se somete al difícil equilibro entre el respeto a la privacidad y la rigurosidad de una labor que es de interés público, esta es el periodismo y sus profesionales, quienes previamente se han formado en universidades de todo el mundo.

Precisamente este artículo pretende mostrar en qué contextos y cómo se aborda el tema de la privacidad en los estudios de periodismo y comunicación. Y para ello, presentamos los resultados de un estudio cuantitativo y cualitativo que analiza 865 materias de las 25 titulaciones que conforman las 13 mejores universidades en comunicación y medios en Europa y Estados Unidos, según los índices internacionales "QS World University Ranking

\section{Abstract}

The right to privacy of citizens and their concern for the protection of their data has been accentuated with the consolidation and massive use of the Internet, where millions of personal references of both direct users and sporadic surfers are increasingly exposed.

But if there is a profession that is subjected to the difficult balance between respect for privacy and the rigorousness of a work that is of public interest, this is journalism and its professionals, who have previously been trained in universities around the world.

This article aims to show in what contexts and how the issue of privacy is addressed in journalism and communication studies. To this end, we present the results of a quantitative and qualitative study that analyzes 865 subjects of the 25 degrees that make up the 13 best universities in communication and media in Europe and the United States, according to the international indexes "QS World University Ranking by Subject".

The conclusions point out that, despite the 
by Subject".

Las conclusiones señalan que, a pesar del interés generado por la ciudadanía, las universidades relegan las enseñanzas relativas a la "privacidad" y la protección de datos a una posición claramente minoritaria en sus planes de estudios. Los necesarios cambios para adecuarse a la nueva realidad deberán asumirlos los futuros profesionales desde sus centros de aprendizaje, porque solo así se logrará el verdadero sentido de su trabajo: el de comprometerse éticamente con la sociedad.

Palabras clave: privacidad, periodismo, universidad, tecnología, deontología. interest generated by the public, universities relegate teaching related to "privacy" and data protection to a clearly minority position in their curricula. The necessary changes to adapt to the new reality must be assumed by future professionals from their learning centers, because this is the only way to achieve the true meaning of their work: that of committing themselves ethically to society.

Keywords: privacy, journalism, university, technology, deontology.

\section{INTRODUCCIÓN}

La definición del concepto de "privacidad" siempre ha ido ligada a las tecnologías disponibles en cada momento (Mendel et al., 2012), pero la "revolución digital" lo ha situado en el centro del debate sobre los derechos que tienen los ciudadanos (Pérez-Tornero, 2016). De hecho, en marzo de 2017, la Oficina del Alto Comisionado de las Naciones Unidas para los Derechos Humanos (ACNUDH) destacó por primera vez, y de forma oficial, el vínculo entre los derechos fundamentales relacionados con la información, el derecho a la privacidad y la libertad de expresión, con el derecho integral al libre desarrollo de la personalidad. En concreto, la resolución A/HRC/34/L. $7^{1}$ reconocía el derecho a la intimidad como aquel que posibilita "el libre desarrollo de la personalidad y, a ese respecto, observaba con preocupación cómo cualquier violación del derecho a la privacidad podría influir en otros como la libertad de expresión y opinión, y la libertad de reunión pacífica y asociación".

En 2020, la cantidad de usuarios conectados a internet en el mundo ya superaba el umbral de los cuatro mil doscientos millones de personas: más de la mitad de la población mundial estaba conectada a la Red de redes, según el informe "Digital 2021. Global Overview Report" (2021). Sin embargo, estar enlazado con la base de datos de información mundial más grande tiene su precio. Jerry Berman y Deirdre Mulligan (1999) ya señalaron a finales del siglo pasado tres factores importantes que han afectado profundamente la "privacidad": el aumento en la web de la creación de datos y el consecuente almacenamiento de grandes cantidades de referencias personales; el registro de casi todas las interacciones que se producen en línea; la globalización del mercado de datos y la capacidad de cualquier persona para recopilar y examinar todas esas informaciones personales; y la falta de mecanismos de control de los datos digitales que existían para proteger los datos analógicos. Pero lo cierto es que todos los estudios posteriores no hacen más que confirmar la creciente preocupación de los ciudadanos por la protección de sus datos. En un artículo reciente, Joseph Turow et al. (2018) muestran que, en Estados Unidos, alrededor del $96 \%$ de los usuarios de Internet teme que se divulguen sus datos personales, como la información de sus tarjetas de crédito o sus números de la seguridad social, lo que podría provocar el robo de identidades y también perjuicios

\footnotetext{
${ }^{1} \mathrm{~A} / \mathrm{HRC} / 34 /$ L.7: "The right to privacy in the digital age", del 17 de marzo de 2017. http://ap.ohchr.org/documents/alldocs.aspx?doc_id=27960
} 
económicos. Solo el $17 \%$ de los estadounidenses creen que su información personal está segura, y alrededor del $50 \%$ de los padres estadounidenses encuestados aseguran haber hablado con sus hijos sobre el robo de la identidad en internet.

Otra fuente de preocupación parece ser la participación de los gobiernos en las actividades online de sus ciudadanos. En Estados Unidos, esos temores se vieron reforzados por la filtración de datos de la Agencia de Seguridad Nacional de los Estados Unidos (NSA) en 2013 y por el posterior espionaje, tanto de ciudadanos estadounidenses como de ciudadanos extranjeros, en todo el mundo. En este sentido, el informe "El estado de la privacidad en la América post Snowden" del Pew Research Center (2016) realizado entre noviembre de 2014 y enero de 2015, muestra cómo el 52\% de los estadounidenses se sentían "muy preocupados" o "bastante preocupados" a este respecto. Sobre los datos y las comunicaciones electrónicas, alrededor del $74 \%$ decía que era muy importante "tener control sobre quién puede obtener información" y el 65\% que era muy importante "vigilar qué información debe recopilarse".

A nivel mundial, el último informe de "KPMG ${ }^{2}$ International" revela que el 55\% de los consumidores encuestados decidió no comprar en internet por cuestiones de falta de privacidad, y menos del $10 \%$ de los consumidores creían tener control sobre cómo las organizaciones administran y usan sus datos personales.

A raíz de todo esto, los gobiernos de todo el mundo están discutiendo y aprobando leyes que buscan proteger la información personal de sus ciudadanos, mientras que, al mismo tiempo, las agencias de seguridad nacional justifican su derecho a acceder a los datos de los usuarios debido a las amenazas terroristas de los últimos años, y empujan a los fabricantes de tecnología a crear códigos especiales que les permitan acceder a sus cuentas en caso de comportamiento sospechoso.

Por otro lado, el Reglamento General Europeo de Protección de Datos (RGPD) -probablemente el más importante en este ámbito- fue aprobado por el Parlamento Europeo y el Consejo de la Unión Europea el 27 de abril de 2016, y entró en vigor el 25 de mayo de 2018. El RGPD (UE) 2016/679 3 es un reglamento de la legislación de la Unión Europea (UE) sobre protección de datos y privacidad para todas las personas dentro de la UE y el Espacio Económico Europeo (EEE), cuyo objetivo principal es otorgar a las personas el control de sus datos personales y simplificar el entorno normativo para los asuntos internacionales, unificando así la normativa dentro de la Unión Europea. Este Reglamento también aborda la exportación de datos personales fuera de las áreas de la UE y del EEE.

El objetivo del RGPD es prevenir una violación de datos personales, lo que se traduciría, en caso de que la infracción fuera por parte de una empresa, en una multa potencial de hasta 20 millones de euros o el $4 \%$ de su facturación anual global.

Muchos analistas en Europa creen que la entrada en vigor de este Reglamento de Protección de Datos está ayudando a mejorar la protección de la privacidad en todo el mundo. Puesto que todos los ciudadanos europeos tienen que aprobar cualquier uso particular de su información, se espera que a las multinacionales les resulte más fácil crear reglas claras y precisas para todos los usuarios, ya sean europeos, ya sean de fuera de ese continente.

\footnotetext{
${ }^{2}$ KPMG: https://home.kpmg.com/sg/en/home/media

${ }^{3}$ www.boe.es/doue/2016/119/L00001-00088.pdf
} 
Sin embargo, como recuerda la UNESCO, el esfuerzo de las autoridades competentes no es suficiente, si los ciudadanos no están equipados con la información y las habilidades necesarias para comprender y hacer valer sus derechos. Por ello, en su Encuesta de 2017: "Survey on Privacy in Media and Information Literacy with Youth Perspectives" (Culver y Grizzle, 2017), la institución insiste en la importancia de la alfabetización mediática, puesto que, al promover una comprensión más profunda de las formas en que los medios y la tecnología permiten o limitan la capacidad para vivir en libertad, resulta ser una herramienta fundamental para la defensa de la privacidad.

\subsection{Privacidad y periodismo}

A partir de este derecho fundamental de la privacidad señalado, lo cierto es que todos los ciudadanos deberían conocerlo, a la vez que utilizarlo, para sentirse protegidos. Pero si existe una profesión en la que el conocimiento continuo actualizado de las condiciones legales (y no legales), con respeto a la privacidad, es de vital importancia hoy más que nunca, esa es, sin duda, el periodismo.

Los periodistas se enfrentan a un difícil equilibrio entre el respeto a la privacidad y la rigurosidad y solidez de sus investigaciones sobre temas que consideran de interés público. El artículo 4 del citado RGPD (UE) 2016/679 define, por ejemplo, la vulneración de datos personales, los data breach, como "la violación de seguridad que implica accidental o ilegalmente la destrucción, pérdida, modificación, divulgación no autorizada o acceso a datos personales transmitidos, almacenados o de otra manera procesados". Por lo tanto, el debate ${ }^{4}$ sobre la pertinencia, implementación y límites de la aplicación de esta legislación en el ejercicio de la profesión periodística acaba de comenzar.

El artículo 85 del Reglamento Europeo también prevé exenciones o derogaciones a favor de la actividad periodística, pero también a favor de la expresión académica, artística y literaria, y cada estado miembro puede ofrecer más exenciones a los periodistas. En general, el tratamiento de datos que realiza el periodista es sustancialmente gratuito: este profesional de la comunicación también puede tratar (y publicar) informaciones sensibles y judiciales sin necesidad de obtener el consentimiento del interesado, siempre que se cumplan dos requisitos: que los datos se hayan recopilado de manera legal y correcta (principio de legalidad); y que la difusión de datos se produzca dentro de los límites de lo esencial (principio de esencialidad) de la información sobre hechos de interés público. Los medios de comunicación, por lo tanto, podrán solicitar una exención si los datos personales que tratan se consideran imprescindibles para su publicación, es decir, si creen que la publicación es de interés público y si su cumplimiento puede ser incompatible con el periodismo (Ruano y Torres, 2016; Lévy, 2007).

En definitiva, sea cual sea el resultado del debate y las aplicaciones y/o cambios en el reglamento, es evidente que, para los periodistas y, en general, para todos los comunicadores, estar adecuadamente preparados y actualizados en materia de privacidad es condición sine qua non para poder ejercer su profesión de una forma óptima, con garantías.

\footnotetext{
${ }^{4}$ Cfr. The Guardian https://www.theguardian.com/commentisfree/2018/jun/10/data-protection-pressfreedom
} 


\section{LA ENSEÑANZA DEL PERIODISMO}

El escenario comunicativo actual, marcado por el impacto de la web 2.0 -también llamada web social- requiere que periodistas tengan una serie de novedosas habilidades, que van desde el dominio de destrezas técnicas para el uso de nuevas herramientas hasta la asimilación de la producción rutinaria de contenidos marcados con una lógica transmediática (Scolari, 2013; Quan-Hasse y Young, 2010; Salaverría, 2016), entre otros aspectos.

El advenimiento del llamado CGU -herramienta de Contenido Generado por el Usuario, a menudo amateur, que es utilizado por periodistas- pone aún más énfasis en temas cruciales como el respeto por los derechos de autor y la privacidad (Autor, 2019).

Vasallo y Fuentes (2000), en el contexto latinoamericano; Westlund (2013), entre otros, en el contexto europeo; y Wenger et al. (2014) en el de Estados Unidos, convergen en la falta de un debate profundo sobre los estudios de comunicación y sobre la necesidad de afrontar, en el ámbito académico, los cambios en el escenario comunicativo con un enfoque sistémico global.

Ya a mediados de la primera década de siglo, la UNESCO (2007) encargó a un grupo de expertos en educación la elaboración de directrices para la enseñanza del periodismo. El estudio concluyó que la formación en periodismo en el ámbito universitario debía organizarse en torno a tres ejes curriculares: a) normas, valores, herramientas, criterios de calidad y prácticas periodísticas; b) los aspectos sociales, culturales, políticos, económicos, legales y éticos del ejercicio del periodismo; y c) Conocimiento del mundo y dificultades intelectuales relacionadas con el periodismo. Esta propuesta nace en una etapa en la que, a pesar de la irrupción de numerosos cibermedios, la reflexión sobre la correcta aplicación de la tecnología en el ámbito periodístico se encuentra en un periodo inicial de desarrollo.

Una década más tarde, en el contexto de una encuesta sobre la formación de periodistas en Brasil, Portugal, Puerto Rico y España, López-García, Rodríguez-Vázquez y Pereira Fariña (2017) destacaron que, a pesar de la mejora en el nivel de formación de los periodistas, se iba a seguir llevando a cabo una revisión permanente de los Planes de estudio, así como el fomento de la educación continua.

Finalmente, en un estudio participado por catorce países de la Unión Europea (Austria, Bélgica, Dinamarca, España, Finlandia, Francia, Grecia, Irlanda, Italia, Países Bajos, Portugal, Reino Unido y Suecia), Díaz del Campo (2013) se concluyó que la inclusión de la ética es esencial en cualquier Plan de estudios de periodismo. El autor destaca que el ejercicio del periodismo requiere de una conciencia ética de estos profesionales que, necesariamente, debe ser proporcionada por la institución educativa.

En 2017, el Report of Predictions que elaboró el Instituto Reuters junto a la Universidad de Oxford, también indicó que, entre los principales desafíos y tendencias del periodismo, la realidad 3D en los teléfonos móviles con fines informativos, el periodismo de datos, la lucha contra las fake news y la privacidad iban a ser fundamentales.

Este nuevo escenario requiere, por tanto, importantes transformaciones: las enseñanzas universitarias de ciencias de la comunicación deben renovar y actualizar sus planes de estudio para adecuarlos a los nuevos perfiles profesionales que demanda el mercado, a las peculiaridades de la situación comunicativa y al nuevo perfil de los usuarios de Internet (Acosta, Costales y Rosales, 2016; López García, 2010; Mellado et al., 2007; Cervi, 2020). Por lo 
tanto, los retos relacionados con la actualización de estos estudios universitarios abarcan todo el proceso educativo, desde los contenidos, pasando por las habilidades y metodologías de enseñanza, hasta llegar a los propios docentes y su manera de enseñar este nuevo escenario que implica profesión y sociedad (Tramullas, 2016; Mellado, 2010).

\section{MÉTODO}

Como hemos señalado anteriormente, son muchos los autores que se han centrado en los estudios del periodismo y sus múltiples disciplinas, pero lo cierto es que no existen investigaciones específicas sobre el papel de la privacidad en la educación periodística. Por ello, nuestro objetivo es comprender en qué contextos y cómo se aborda el tema de la privacidad en la educación universitaria del periodismo y, en general, de la propia en comunicación. Y para ello, nos centraremos en las mejores universidades del mundo.

El estudio se basa en el prestigioso "QS World University Ranking by Subject", que clasifica las universidades -y sus materias- a través de una serie de indicadores como la reputación académica (a través de una encuesta a 70.000 académicos), la reputación profesional (encuesta a 30.000 profesionales expertos en la materia), la relación profesor/alumno, el número de citas por facultad, y la relación internacional entre alumnos y profesores.

Inspirados por los estudios de Cervi (2017); Cervi, Pérez-Tornero y Tejedor (2020), hemos seleccionado las 10 mejores universidades del mundo en el área de "Comunicación y medios", incluyendo todas las titulaciones relacionadas con la comunicación y/o el periodismo.

Las universidades seleccionadas (y sus rankings) son: Universidad Southern California, Estados Unidos (1), Universidad de Ámsterdam, Holanda (2), London School of Economics and Political Science, Gran Bretaña (3), Universidad de Stanford, Estados Unidos (4), Universidad de Wisconsin-Madison, Estados Unidos (5), Universidad de California-Berkeley, Estados Unidos (= 6), Universidad de Texas, Austin, Estados Unidos (=6), Universidad Goldsmiths, de Inglaterra (8), Universidad de California-Los Ángeles (UCLA) (9) y Universidad de California-Los Ángeles Pennsylvania, Estados Unidos (10). La Universidad de Texas y la London School of Economics han sido excluidas de la muestra, ya que no ofrecen títulos en estas disciplinas, solo maestrías y doctorados.

La muestra inicial estuvo compuesta casi en su totalidad por universidades estadounidense. Por este motivo, y para obtener una muestra más heterogénea, también se sumaron las primeras 15 universidades europeas presentes en la última actualización del ranking del año 2018. Las universidades seleccionadas fueron: Universidad de Ámsterdam, Países Bajos (1), London School of Economy and Political Science, Gran Bretaña (3), Goldsmith, Universidad de Londres, Gran Bretaña (9), Universidad de Cardiff, Gran Bretaña (23), King's College, Gran Bretaña (24), Universidad de Zurich, Suiza (30), Universidad de Arhus, Dinamarca (= 35), Ludwig-Maximilians-Universität de Munich, Alemania (=35), Universidad de Leeds, Gran Bretaña (39), Universidad de Westminster, Gran Gran Bretaña (40), Universidad de Loughborough, Gran Bretaña (41), Universidad de Helsinki, Finlandia (43), Universidad de Viena, Austria (44), Universidad de Navarra, España (49) y Universidad de Leicester, Gran Bretaña (50).

Tanto la London School of Economics and Political Science como el King's College no ofrecen cursos de grado, solo especializaciones de posgrado, por lo que han sido excluidas. Además, 
por razones de comprensión idiomática común, se han excluido las universidades que no ofrecen cursos en inglés: por este motivo, de las universidades de habla inglesa, solo se han considerado las Universidades de Ámsterdam y Navarra.

Muchas universidades ofrecen más de una titulación en este campo; así pues, las 25 titulaciones analizadas corresponden a 13 campus universitarios (ver la tabla 1).

En cuanto a la metodología utilizada para responder a las preguntas de investigación, se ha desarrollado un corpus de análisis en el que se han recogido los siguientes aspectos: a) Estructura; b) Objetivos y competencias de las carreras; c) Distribución de asignaturas; d) Presencia o ausencia de asignaturas específicas sobre ética o derecho de la información; e) Cantidad y tipo de temas en los que se aborda el tema de la privacidad; f) Presencia de temas específicos de privacidad.

De las 865 asignaturas ofertadas en las carreras en cuestión (en el caso de Estados Unidos, no se incluyen las optativas de otras disciplinas), se han seleccionado todas aquellas materias en cuyos títulos se menciona explícitamente la palabra "privacidad", además de todas las asignaturas de Derecho y/o Ética de la Información y Políticas de comunicación.

A través de un análisis de contenido cuantitativo, se han escogido aquellas carreras que realmente hablan de privacidad $y$, posteriormente, han sido sometidas a un análisis de contenido cualitativo para comprender desde qué punto de vista y cómo afrontan esta temática.

Tabla 1: Universidades y titulaciones

\begin{tabular}{|l|c|c|}
\hline \multicolumn{1}{|c|}{ Titulación } & Universidad & País \\
\hline Comunicación & Universidad de Stanford & EE.UU. \\
\hline Comunicación & Universidad de California-Berkeley & EE.UU. \\
\hline Comunicación & Universidad de Wisconsin-Madison & EE.UU. \\
\hline Periodismo & Universidad de Wisconsin-Madison & EE.UU. \\
\hline Comunicación & Universidad de California-Los Ángeles - UCLA & EE.UU. \\
\hline Comunicación & Universidad de Pensilvania & EE.UU. \\
\hline Comunicación & Universidad Southern California & EE.UU. \\
\hline Periodismo & Universidad Southern California & EE.UU. \\
\hline $\begin{array}{l}\text { Ciencias de la } \\
\text { Comunicación }\end{array}$ & Universidad de Ámsterdam & Holanda \\
\hline $\begin{array}{l}\text { Información y Medios } \\
\text { de Comunicación }\end{array}$ & Universidad de Ámsterdam & Holanda \\
\hline Comunicación y Medios & Goldsmiths, Universidad de Londres & Reino Unido \\
\hline Periodismo & Goldsmiths, Universidad de Londres & Reino Unido \\
\hline
\end{tabular}




\begin{tabular}{|c|c|c|}
\hline $\begin{array}{l}\text { Periodismo, Medios de } \\
\text { Comunicación y } \\
\text { Literatura inglesa }\end{array}$ & Universidad de Cardiff & Reino Unido \\
\hline $\begin{array}{l}\text { Periodismo, } \\
\text { Comunicación y } \\
\text { Sociología }\end{array}$ & Universidad de Cardiff & Reino Unido \\
\hline $\begin{array}{l}\text { Periodismo, } \\
\text { Comunicación y Política }\end{array}$ & Universidad de Cardiff & Reino Unido \\
\hline Medios y Comunicación & Universidad de Cardiff & Reino Unido \\
\hline Medios y Comunicación & Universidad de Leeds & Reino Unido \\
\hline Periodismo & Universidad de Leeds & Reino Unido \\
\hline Medios digitales & Universidad de Leeds & Reino Unido \\
\hline $\begin{array}{l}\text { Medios digitales y } \\
\text { Comunicación }\end{array}$ & Universidad de Westminster- Londres & Reino Unido \\
\hline Periodismo & Universidad de Westminster- Londres & Reino Unido \\
\hline $\begin{array}{l}\text { Comunicación y } \\
\text { Estudios de Medios }\end{array}$ & Universidad de Loughborough & Reino Unido \\
\hline Periodismo (bilingüe) & Universidad de Navarra & España \\
\hline Periodismo & Universidad de Leicester & Reino Unido \\
\hline Medios y Comunicación & Universidad de Leicester & Reino Unido \\
\hline
\end{tabular}

Fuente: elaboración propia.

\section{RESULTADOS}

\subsection{Estructura de las carreras}

En este apartado describiremos brevemente la estructura de las titulaciones que ofrecen las universidades de la muestra, con el fin de contextualizar e identificar los elementos fundamentales para la comparativa. De hecho, es importante subrayar que cada país cuenta con diferencias notables en su planificación curricular con respecto a otros territorios. El sistema universitario estadounidense, por ejemplo, difiere mucho del europeo en general.

Aunque hay más de 4.500 instituciones de educación superior acreditadas, lo primero que hay que señalar es que no existe un Plan de Estudio genérico, ni una agencia o institución de control central, ya que todo se gestiona en el ámbito de cada estado de forma individual. El sistema de créditos, por tanto, al no estar centralizado, puede ser muy diferente de una universidad a otra, al igual que los Planes de estudio y el marco básico de las titulaciones. Además, a diferencia de muchos países europeos, en Estados Unidos no existen las facultades, por lo que los estudiantes cursan durante los dos primeros años diferentes disciplinas como literatura, arte, historia, ciencia, etc., con el fin de crear un perfil de cultura general. Al final del segundo año (muchas universidades prohíben expresamente hacerlo antes), los estudiantes pueden decidir en qué disciplina enfocarse. Una especialización académica es, por lo tanto, la disciplina curricular en la que el estudiante decide encaminarse. Después, los estudiantes 
pueden elegir, aunque no necesariamente, tres años de estudio como segunda especialización académica, que puede ser significativamente diferente de la principal.

Finalmente, en cuanto al tipo de asignaturas, el estudio revela que las universidades estadounidenses tienden a ser mucho más flexibles y limitan el número de materias obligatorias con el fin de ofrecer al estudiante la posibilidad de construir un currículo ad hoc en función de sus intereses.

La trayectoria académica de los estudiantes estadounidenses, por lo tanto, puede ser muy heterogénea, dadas las amplias posibilidades de cursar asignaturas fuera de su carrera. Por ello, a efectos de una adecuada comparativa, solo se consideran materias relacionadas con las especializaciones.

Las universidades británicas, a pesar de que pertenecen formalmente al Espacio Europeo de Educación Superior, no aplican todos los estándares, especialmente en términos de recuento de créditos; sin embargo, a nivel estructural son muy similares a las universidades europeas. De hecho, trabajan un Bachillerato de Artes (BA) o de Ciencias (BC) con una duración de tres o cuatro años y son el equivalente a un título en los principales países europeos. El resto de las universidades europeas analizadas siguen el sistema europeo de créditos ECTS y se adaptan al "Plan Bolonia", desde una perspectiva amplia para que el análisis se pueda llevar a cabo. De hecho, Holanda y España utilizan sistemas similares, pero conviene hacer algunas aclaraciones. En Holanda, la educación superior se caracteriza por tener un sistema mixto: por un lado, existen universidades de ciencias aplicadas o profesionales donde se imparte una docencia más práctica, con una duración de 4 años. Por otro lado, están las universidades clásicas que imparten, entre otras, los estudios de periodismo y comunicación, además de realizar actividades de investigación. En estos casos, el Grado tiene una duración de 3 años y aglutina 180 créditos ECTS (European Credit Transfer System). En España, la mayoría de las universidades no aplican el sistema "3 + 1" ni "3 + 2", aplicando un "4 + 1": la titulación tiene una duración de cuatro años y se puede complementar con un año de Máster que da acceso al doctorado.

\subsection{Objetivos y competencias}

El análisis de los 25 planes de estudio de las licenciaturas en periodismo y/o comunicación de las distintas universidades seleccionadas permite identificar los 9 objetivos didácticos más comunes, enumerados seguidamente de mayor a menor importancia:

- Cultura digital: estudia las particularidades que definen la llamada "Cultura digital" para promover la comprensión de las transformaciones que la tecnología ha generado en el escenario de la comunicación.

- Fundamentos de la comunicación: analiza las bases de los procesos de comunicación a nivel conceptual y su evolución en el tiempo.

- Producción de contenidos: desarrolla habilidades para la concepción y generación de contenidos informativos de diversa índole (tanto formatos como medios).

- Industria de los medios: explora las características que definen la estructura y las políticas de comunicación de ámbito nacional e internacional. 
- Aspecto crítico: Asume habilidades críticas y reflexivas relacionadas con diferentes mensajes multimedia y rutinas de producción.

- Creatividad: desarrolla la capacidad de concebir mensajes, formatos y proyectos de comunicación originales que exploten las posibilidades de cada medio, sector o área temática.

- Mirada humanista: Promueve una asimilación básica de diferentes disciplinas o áreas como la filosofía, la historia o la antropología, entre otras.

- Versatilidad: Explora la capacidad de aplicación de las rutinas de producción de diferentes soportes con solvencia, rigor y profesionalidad.

- Efectos de la comunicación: Tiene como finalidad comprender los efectos de la comunicación y sus procesos en las personas y grupos sociales.

Entender, por lo tanto, las transformaciones que introduce la cultura digital en el escenario comunicativo parece ser un objetivo recurrente. Si nos centramos en las competencias, el estudio de los currículos seleccionados nos permite identificar un grupo de 11 competencias comunes. Se trata de conocimientos, talentos y actitudes esenciales que incluyen tanto habilidades básicas (también llamadas clave, esenciales o fundamentales) como específicas, más de corte profesional y académico. Las habilidades comunicativas, en general, junto con la adquisición de una actitud de autorreflexión, ocupan un peso importante en los currículos analizados. Y hay que insistir en la necesidad de conocer los fundamentos de los intercambios comunicativos y en la capacidad de poner en práctica las técnicas de la profesión siguiendo su ética profesional. En concreto, la competencia que se repite varias veces en el conjunto de planes de estudio es precisamente la capacidad de actuar de forma crítica, analítica y ética. Dado el aparente énfasis puesto en la ética, uno esperaría una presencia importante de los problemas que aporta el tema de la "privacidad", pero los datos refutan abiertamente esta suposición.

\subsection{Materias obligatorias}

Las asignaturas obligatorias son las llamadas "fundamentales", consideradas los pilares del conocimiento. Por ello, resulta especialmente interesante comenzar nuestro análisis a partir de ellas.

Como se puede observar en el Gráfico 1, el 29\% corresponde a temas relacionados con la Teoría, Estructura e Historia de la comunicación, que junto a las Metodologías de investigación (con el $19 \%$ del total) y los conocimientos básicos (16\%), representan más de la mitad de las materias obligatorias. Estos datos indican que las asignaturas obligatorias apuestan por consolidar una formación fundamentalmente teórica. 


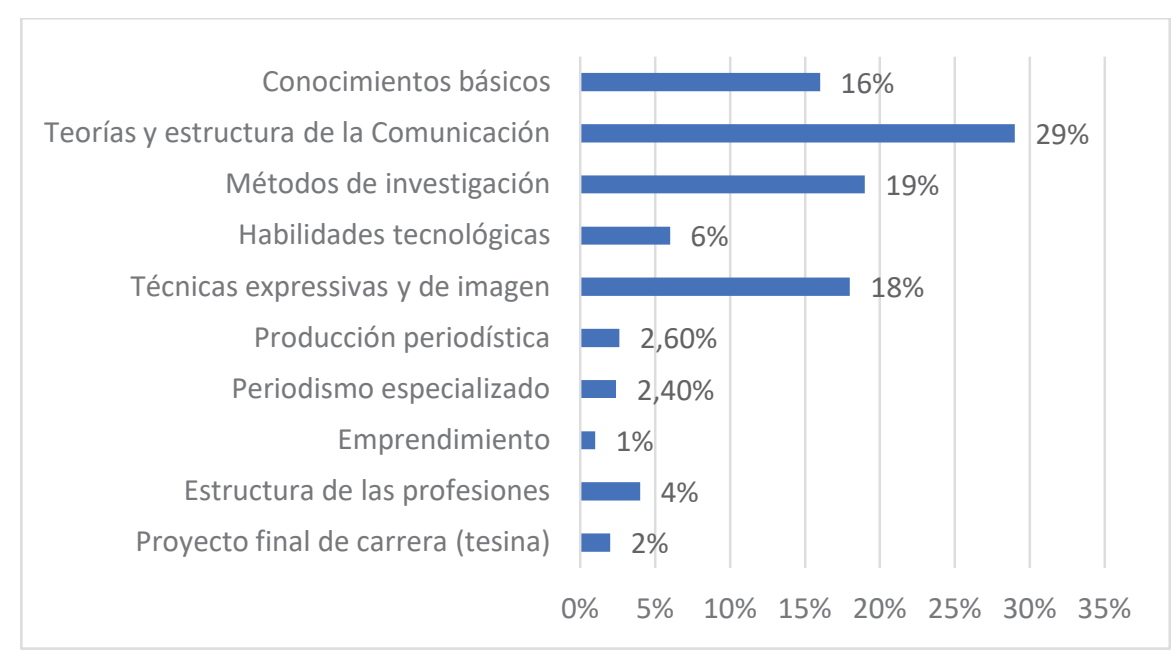

Fuente: elaboración propia.

En el área temática "Teorías, Estructuras e Historia de la comunicación", encontramos asignaturas relativas a la Teoría de la comunicación, que tienen como objetivo proporcionar capacidades comunicativas a partir del intercambio de información; por ejemplo: Introducción a la Comunicación o Comunicación en las Ciencias Sociales. Pero sobre todo que proveen de temas que tratan de abarcar el panorama mediático desde una perspectiva más sociológica, como es el caso de Medios, Cultura y Sociedad, presentes, con diferentes nombres, en todas las universidades.

Las cuestiones relativas al marco legal, Las Políticas de Comunicación y/o La Ética y la Deontología de la Comunicación (Ética de la Comunicación, Derecho de los Medios de Comunicación o Ética de los Medios de Comunicación) también entran dentro de esta área temática.

Como muestra el Gráfico 2, hay materias como Políticas de Derecho, Ética y Comunicación, que obtienen una presencia mínima en los Planes de estudio. De hecho, de las 25 titulaciones examinadas, solo 16 asignaturas -que son obligatorias y representan $1,8 \%$ del total de asignaturas- tratan de Ética/Deontología o Derecho de los Medios. Esto significa que no todas las titulaciones en periodismo o comunicación consideran necesario impartir formación en Derecho de la Comunicación a futuros periodistas o profesionales de la comunicación. Además, solo la Universidad de Berkeley cuenta entre sus asignaturas obligatorias: "Privacidad en la era digital", una materia específica dedicado al objeto de este estudio.

Gráfico 2: Distribución de asignaturas del área temática: "Teorías, Estructuras e Historia de la Comunicación" 


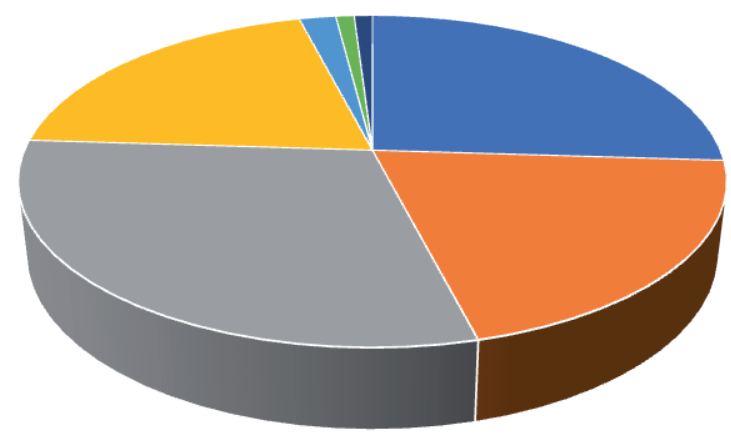

$$
\begin{array}{ll}
\text { - Teorías de la Comunicación } & \text { - Estructuras de la Comunicación } \\
\text { - Medios de Comunicación y Sociedad } & \text { - Internet y Sociedad } \\
\text { - Ética de la Comunicación } & \text { - Derecho de la Comunicación } \\
\text { - Políticas de la Comunicación } &
\end{array}
$$

Fuente: elaboración propia.

\subsection{Materias optativas}

Observando la distribución de las asignaturas optativas, representada por el Gráfico 3, podemos ver cómo los "Conocimientos Básicos" (Historia, Sociología, Antropología, etc.) son preponderantes. Como ya se ha comentado anteriormente, los datos representan en su mayoría a las universidades estadounidenses de la muestra, donde se favorece el estudio de este tipo de asignaturas genéricas.

\section{Gráfico 3: Materias optativas}

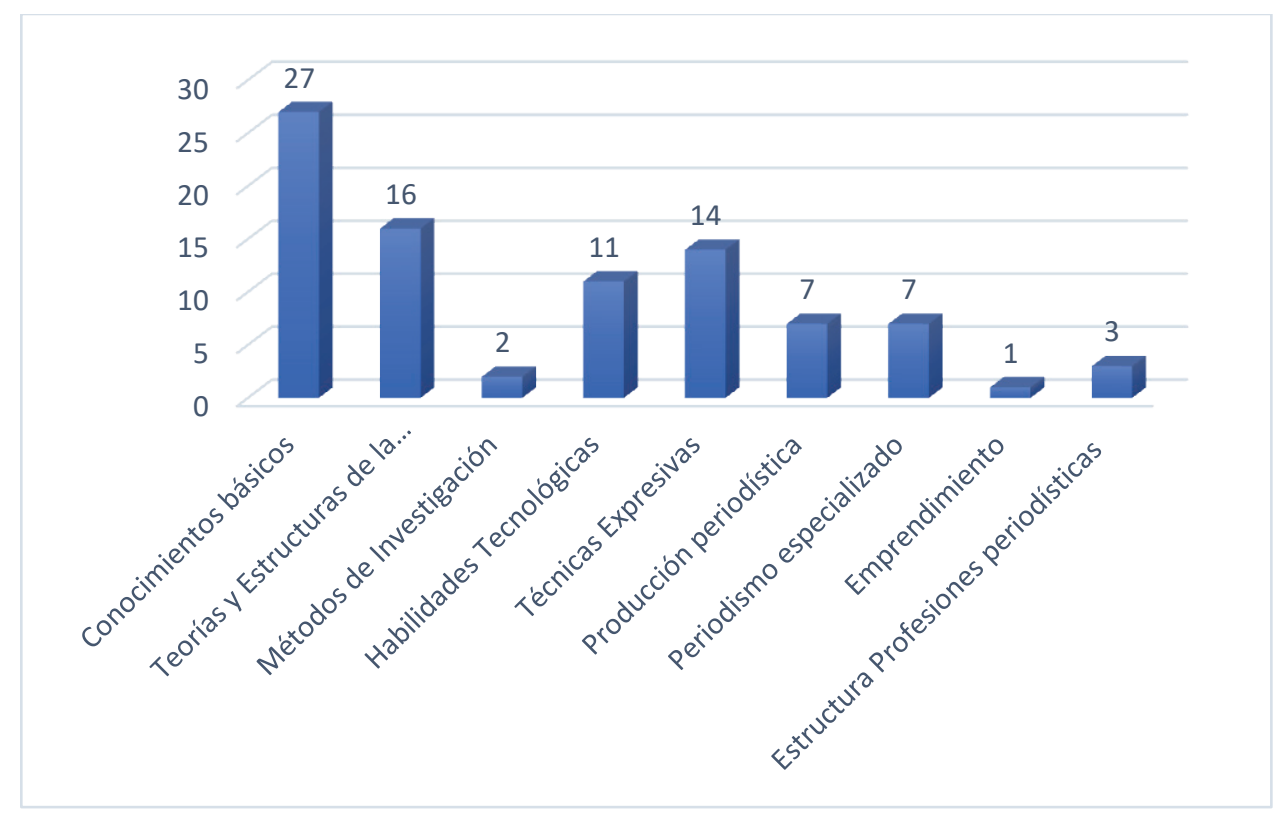

Fuente: elaboración propia.

Los resultados no difieren de los propios de las asignaturas obligatorias: en total las materias que tratan de ética o derecho de la información representan el 2,5\%.

\subsection{Tipo de materias que tratan el tema de la "privacidad"}


Del total de asignaturas, se seleccionan 34 materias, de las que 15 son obligatorias y 27 son optativas. Se descartan 5 de ellas porque tratan el "Derecho" de una forma demasiado genérica (Freedom of Speech como se dice en EE.UU.) y no dedican ningún espacio a la temática de esta investigación. Por lo tanto, de la muestra seleccionada solo el 3\% del total trata la "privacidad".

Si nos fijamos en el tipo de asignatura, como se muestra en el Gráfico 4, la "privacidad" es tratada casi en un $50 \%$ de las materias de derecho, impartidas por abogados; seguidos de asignaturas de ética, impartidas por profesores con diferentes perfiles académicos (periodistas, filósofos, etc.); y por una amalgama de otras disciplinas que van desde la sociología hasta el análisis de políticas.

\section{Gráfico 4: Distribución temática de las materias relacionadas con la privacidad}

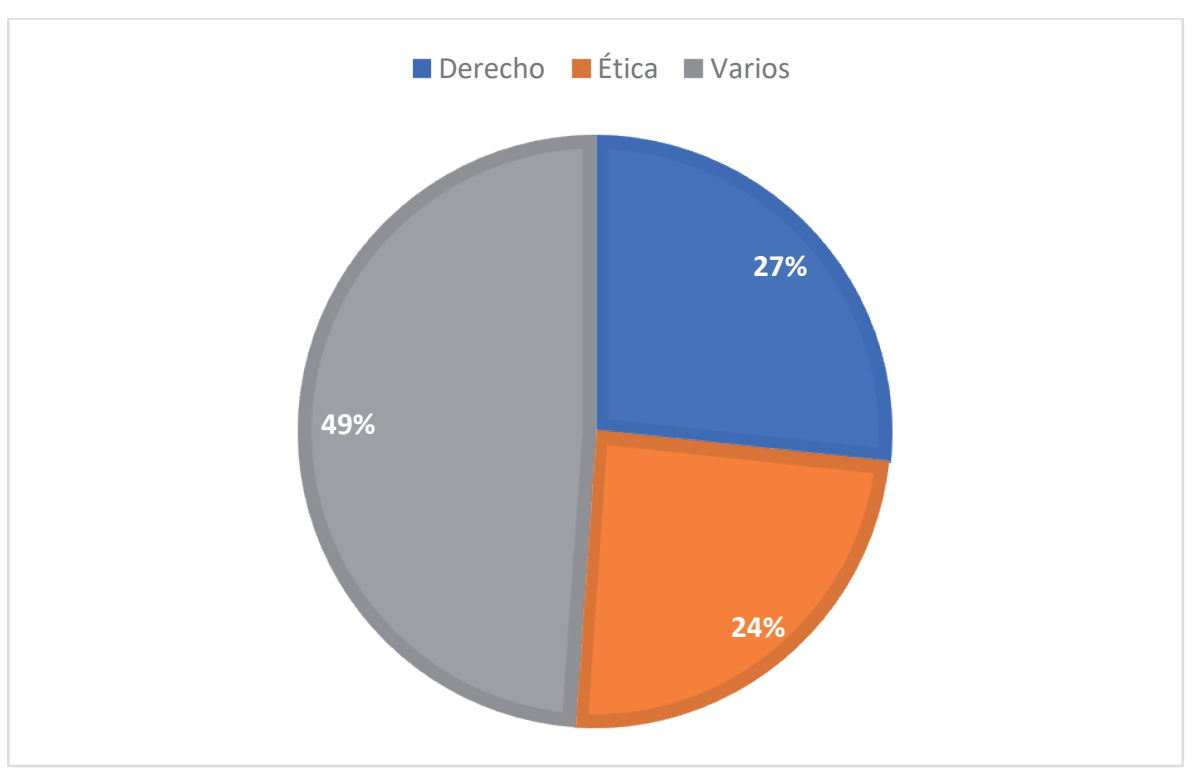

Fuente: elaboración propia.

\subsection{Contenidos}

El análisis cualitativo de los contenidos nos permite establecer cómo las diferentes materias abordan el tema de la "privacidad". En primer lugar, las asignaturas mixtas de ética y deontología (por ejemplo, Ética de los Medios y Derecho) se centran, en su mayor parte, en los derechos y responsabilidades de los periodistas, mientras que las de ética se centran en el debate filosófico, más que en la práctica periodística.

En general, las asignaturas de "derecho" tienden a abordar los principales problemas del Derecho de la Información y la Comunicación, la Organización -cuando esta existe- de la Profesión Periodística, y el Sistema de Medios y las Autoridades Reguladoras.

Todas las materias asociadas al Derecho (medios, información, internet) cuentan con un módulo dedicado a la privacidad, generalmente relacionado con los problemas legales que plantean las nuevas tecnologías de la información y la comunicación: libertad de comunicación y expresión a través de internet, responsabilidad de los operadores, herramientas de regulación de la red, procesamiento de datos personales... 
En estos casos, el enfoque de la privacidad desde un punto de vista de contenido evidencia las diferencias tradicionales entre las dos culturas occidentales de privacidad. De hecho, los estadounidenses tienden a considerarlo una cuestión de libertad, una protección contra la intrusión del Estado, estrechamente ligada a los derechos de propiedad privada y la libertad de expresión $\mathrm{y}$, de hecho, en las asignaturas se da mucha más importancia al concepto de "vigilancia" y al derecho del ciudadano a no ser "espiado" por el Estado, mientras que en los países europeos -especialmente España- relacionan la intimidad con la dignidad, idea que se origina en el concepto histórico del honor, muchas veces ligado a la protección frente a la intromisión de los medios de comunicación en la vida privada de la gente y su derecho al olvido. Incluso en las tres materias específicas sobre privacidad es posible detectar diferencias sustanciales: en ambas materias de las universidades estadounidenses se centran, de hecho, en el concepto de "vigilancia" por parte de los gobiernos.

Por otro lado, la asignatura obligatoria: Privacidad en la Era Digital, ofrecida en la Universidad de Berkeley, examina los problemas de privacidad en la sociedad contemporánea, con especial énfasis en cómo la privacidad se ve afectada por el cambio tecnológico. Después de una Introducción al Orden Legal Estadounidense y de estudiar Los fundamentos Teóricos de la Ley de Privacidad, el concepto de "privacidad" se analiza en el contexto de las investigaciones de seguridad nacional, registros y bases de datos gubernamentales, noticias, bases de datos comerciales, etc. El programa dedica un módulo completo a la reflexión sobre cómo y por qué la regulación de la privacidad puede limitar la Primera Enmienda y viceversa.

\section{DISCUSIÓN Y CONCLUSIONES}

De las 25 titulaciones analizadas, los resultados muestran que solo el $3 \%$ de las asignaturas aplican de alguna manera la "privacidad" en sus programas, y solo el 0,2\% de las asignaturas precisamente 3 de 865- están dedicadas específicamente a esta temática.

Aunque la privacidad y su protección están en el centro de las preocupaciones de gobiernos y ciudadanos, y a pesar de que la UNESCO y otras instituciones apelan a la educación, es evidente que las mejores universidades con estudios de Comunicación y Periodismo analizadas parecen tener una opinión diferente $y$, sobre todo, un enfoque distinto, ya que relegan las enseñanzas relativas a la privacidad y a la protección de datos a una posición claramente minoritaria.

Por ello, podemos afirmar que nuestro estudio corrobora las reflexiones generales de Singh et al. (2015) y Salaverría (2016), que subrayan la necesidad de reformular los planes de estudio de las facultades de periodismo y comunicación con el fin de dotar a los estudiantes de las herramientas necesarias para afrontar las transformaciones, en particular las tecnológicas, que deben asumir después cuando pasen a ejercer la profesión periodística. Es decir, son las Facultades de Periodismo las que deben adaptarse al cambio tecnológico, dotando a los estudiantes de las habilidades necesarias para utilizar las últimas técnicas, pero sobre todo deben enseñarles a analizar, contextualizar y reflexionar sobre la esencia de los cambios introducidos por esa tecnología.

En la llamada Sociedad de la Información, en la que la propia información se ha convertido en el bien fundamental, la enseñanza de temas de comunicación no puede ignorar una cuestión tan crucial como son los datos y su protección. 
Con el advenimiento del periodismo ciudadano -entendido como un conjunto de procesos con los que el público pasa de ser un lector pasivo a participar en una gran conversación social emergente (Gilmor, 2004), y la consecuente (e inevitable) tendencia hacia el periodismo colaborativo y deliberativo-, el periodista se está convirtiendo en el moderador de esta conversación. Conocer los límites y respetarlos, por lo tanto, se vuelve fundamental para que esta "conversación" sea productiva.

\section{Referencias}

Acosta, M.; Costales, Z.; Rosales, B. (2016): Formación por competencias profesionales en la carrera de Periodismo. Rev. Cubana Edu. Superior, vol.35, no 1, pp. 75-84.

Berman, J.; Mulligan, D. (1999). Privacy in the Digital Age: Work in Progress, Nova Law Review, vol. 23, nํ. 2.

Cervi, L. (2017) Citizen Journalism and User Generated Content in Mainstream Media. new dialogic form of communication, user-engagement technique or free labor exploitation? Revista de Comunicaçao Dialogica, (1), 120-141. https://doi.org/10.12957/rcd.2019.41871

Cervi, L.; Pérez Tornero, J.M.; Tejedor, S. (2020). The Challenge of Teaching Mobile Journalism through MOOCs: A Case Study. Sustainability (12), 5307. https://doi.org/10.3390/su12135307

Cervi, L. (2020). Exclusionary Populism and Islamophobia: A Comparative Analysis of Italy and Spain. Religions, 11, 516.

Culver, S. H.; Grizzle, A. (2017). Survey on Privacy in Media and Information Literacy with youth perspectives. USA, University: UNESCO. http://unesdoc.unesco.org/images/0025/002589/258993e.pdf

Díaz del Campo-Lozano, J. (2013). El lugar de la Ética en la formación del periodista. Un estudio de la situación en la Unión Europea. Cuadernos.Info, 33, pp. 113-120.

Digital 2021. Global Overview Report (2021): https://wearesocial.com/digital-2021

Gilmor, D. (2004). We the Media. Sebastopol. California: O'Reilly.

Lévy, L. (2007). Cibercultura: la cultura de la sociedad digital. Barcelona: Anthropos.

López García, X. (2010). La formación de los periodistas en el siglo XXI en Brasil, España, Portugal y Puerto Rico. Revista Latina de Comunicación Social, 65. La Laguna (Tenerife): Universidad de La Laguna, pp 231- 243.

López-García, X.; Rodríguez-Vázquez, A.; Pereira-Fariña, X. (2017). Technological Skills and New Professional Profiles: Present Challenges for Journalism. Comunicar, 53, pp. 81-90.

Mellado, C.; Simon, J.; Barría, S.; Enríquez, J. (2007). Investigación de perfiles profesionales en periodismo y comunicación para una actualización curricular permanente. Zer, 23, 2007, p.139164.

Mellado, C. (2010). La voz de la academia: Reflexiones sobre periodismo y comunicación. Signo y Pensamiento, vol. XXIX, núm. 56, pp. 274-287.

Mendel, T.; Puddephatt, A.; Wagner, B.; Hawtin, D.; Torres, N. (2012). Global survey on internet privacy and freedom of expression. Unesco. http://unesdoc.unesco.org/images/0021/002182/218273e.pdf

NiemanLab (2017): http://www.niemanlab.org/collection/predictions-2017/ (01-08-2017)

Pérez Tornero, J.M. (2016): Promoting Digital Literacy. Informe Final EAC/76/03. Comprender la alfabetización digital. Barcelona: Gabinete de Comunicación y Educación, Universitat Autònoma 
de

Barcelona.

http://www.gabinetecomunicacionyeducacion.com/sites/default/files/field/adjuntos/compren der_dl.pdf (01-08-2017)

Pew Research Center (2016). "The state of privacy in post-Snowden America". http://www.pewresearch.org/fact-tank/2016/09/21/the-state-of-privacy-in-america/

Quan-Haase, A; Young, A. L. (2010). Uses and gratifications of social media: A comparison of Facebook and instant messaging. Bulletin of Science, Technology \& Society, 30(5), pp. 350-361.

Reuters Institute (2017): Journalism, Media and Technology Trends and Predictions 2017. http://reutersinstitute.politics.ox.ac.uk/our-research/journalism-media-and-technology-trendsand-predictions-2017 (01-08-2017)

Ruano, I. E.; Torres, A. E. (2016). Comunicación e interacción por el uso de dispositivos tecnológicos y redes sociales virtuales en estudiantes universitarios. Revista Ibérica de Sistemas e Tecnologias de Información, 19, pp. 15-31

Salaverría, R. (2016). Redefinir al comunicador. El profesional de la información, v. 25, n. 2, pp. 163-167.

Scolari, C.A. (2013). Media Evolution. Emergence, Dominance, Survival and Extinction in the Media Ecology. International Journal of Communication 7, pp. 1418-1441.

Singh, J.; Grizzle, A.; Joan Yee, S. \& Hope Culver, S. (Eds.) (2015). Media and Information Literacy for the Sustainable Development Goals. MILID Yearbook 2015. Gothenburg: The International Clearinghouse on Children, Youth and Media; Nordicom. http://www.nordicom.gu.se/sites/default/files/publikationer-helapdf/milid_yearbook_2015.pdf

Tramullas. J. (2016). Hannibal ad-portas o los futuros perfiles profesionales de la información. El profesional de la información, v. 25, n. 2, pp. 157-162.

Turow, J.; Hennessy, M. \& Draper, N. (2018). Persistent Misperceptions: Americans' Misplaced Confidence in Privacy Policies, 2003-2015. Journal of Broadcasting \& Electronic Media, 62:3, 461-478.

Unesco (2007): Colección de la UNESCO sobre los estudios de periodismo Plan modelo de estudios de periodismo. 2007. París.

Vasallo, M.; Fuentes, R. (comps.) (2000). Comunicación. Campo y objeto de estudio. Perspectivas reflexivas latinoamericanas. México D. F.: Iteso, Universidad Autónoma de Aguascalientes, Universidad de Colima, Universidad de Guadalajara.

Wenger, D., Owens, L., Thompson, P. (2014). Help Wanted: Mobile Journalism Skills Required b Top U.S. News Companies. Electronic News, vol 8(2), 138-149.

Westlund, O. (2013). Mobile News: A review and model of journalism in an age of mobile media. Digital Journalism, Vol. 1, No. 1, 2013, 6-26.

\section{Semblanza de los autores}

Laura Cervi es Profesora Serra Húnter del Departamento de Periodismo y Ciencias de la Comunicación de la UAB. Doctora en Ciencia Política por la Universitá di Pavia, Italia y por la UAB. Es miembro del grupo de investigación Gabinete de Comunicación y Educación, dirigido por el catedrático Dr. José Manuel Pérez Tornero en la UAB y coordinadora académica del Máster en Gestión de la Comunicación Política y Electoral del Departamento de Periodismo y Ciencias de la Comunicación de la UAB. Ha participado en diversos proyectos internacionales y 
ha sido, junto al Dr. José Manuel Pérez Tornero, Investigadora Principal del proyecto europeo Y-NEX, European Youth News Exchange, (2015-2017). En el año 2014 fue investigadora visitante en la Newberry Library de Chicago (EEUU).

Carles Marín Lladó es Profesor titular de Periodismo Audiovisual en la Universidad Rey Juan Carlos (URJC) desde 2003. Se ha especializado en la información televisiva y radiofónica a partir de su análisis discursivo. Desde 2012 dirige el Máster en Reporterismo de Televisión en la misma universidad. Ha publicado una decena de libros y numerosos artículos sobre reporterismo e informativos audiovisuales, y cuenta con una larga trayectoria en TV y radio como creador de formatos, director, guionista y reportero tanto de programas informativos como de infoentretenimiento. Ha sido vicerrector y vicedecano de la URJC y directivo de la Academia de las Ciencias y las Artes de Televisión (AcademiaTV). 